प\$ SXQUAV8 QIYHWWURV, 2021: 11(2), abril-junio ISSN: 2304-0335 DOI: https://doi.org/10.17162/au.v11i2.634

\title{
Esquemas desadaptativos tempranos y bienestar psicológico en pacientes que acuden a consulta externa en una Institución Prestadora de Servicios de Salud - IPRESS, Lima, 2020
}

\author{
Early maladaptive schemes and psychological well-being in patients who attend an \\ outpatient consultation at a Health Service Provider Institution - \\ IPRESS, Lima, 2020.
}

\author{
Segundo Alamiro Monteza Vásquez ${ }^{1 \mathrm{a}}$ y Daniel Noboru Yogui Takaesu² \\ Universidad Ricardo Palma, Lima, Perú ${ }^{1}$ \\ Universidad Tecnológica del Perú, Lima, Perú \\ ORCID ID: https://orcid.org/0000-0002-9240-535X ${ }^{1}$ \\ ORCID ID: https://orcid.org/0000-0002-6478-9093²
}

Recibido: 19 de octubre de 2020

Aceptado: 05 de enero de 2021

\begin{abstract}
Resumen
En el presente estudio se planteó como objetivo determinar la relación entre los esquemas desadaptativos tempranos y el bienestar psicológico de los pacientes que acuden a consulta externa en una Institución Prestadora de Servicios de Salud - IPRESS, Lima, 2020. La investigación se desarrolló bajo un enfoque cuantitativo, diseño no experimental y un alcance correlacional; en la cual participaron 120 pacientes (ambos sexos) que acudieron al servicio consulto externa de una Institución Prestadora de Servicios de Salud - IPRESS de la ciudad de Lima. La recolección de datos se realizó utilizando dos instrumentos: Escala de esquemas de Young (YSQ-SF) y la Escala de Bienestar psicológico Bieps-A. En el estudio se concluyó que los esquemas desadaptativos tempranos presentan una relación significativa $(\mathrm{p}=0,000)$ e inversa $(\mathrm{r}=-0,764)$ con el bienestar psicológico en pacientes que acuden a consulta externa en una IPRESS, Lima, 2020. Es decir a mayor presencia de esquemas desadaptativos, los pacientes tienden a experimentar menores niveles de bienestar psicológico.
\end{abstract}

Palabras clave: Esquemas desadaptativos, bienestar psicológico, necesidades emocionales tempranas, pacientes de consulta externa.

${ }^{a}$ Correspondencia al autor:

E.mail: smontezav@gmail.com 


\begin{abstract}
The objective of this study was to determine the relationship between early maladaptive schemes and the psychological well-being of patients who attend outpatient consultation at a Health Service Provider Institution - IPRESS, Lima, 2020. The research was developed under an approach quantitative, non-experimental design and a correlational scope; In which 120 patients participated (both sexes) who attended the external consulting service of an Institution Provider of Health Services - IPRESS in the city of Lima. Data collection was carried out using two instruments: Young's Scheme Scale (YSQ-SF) and the Bieps-A Psychological Well-being Scale. The study concluded that early maladaptive schemes present a significant $(\mathrm{p}=0.000)$ and inverse $(\mathrm{r}=-0.764)$ relationship with psychological well-being in patients who attend an outpatient clinic at an IPRESS, Lima, 2020. That is, the higher In the presence of maladaptive schemes, patients tend to experience lower levels of psychological well-being.
\end{abstract}

Keywords: Maladaptive schemes, psychological well-being, early emotional needs, outpatient patients

\title{
Introducción
}

Desde etapas tempranas, el ser humano interactúa con el entorno orientado a satisfacer sus necesidades (fisiológicas, emocionales, sociales), estando expuesto a diferentes experiencias dentro de sus grupos de pertenencia. En esta etapa vital, cuando las personas están expuestas a experiencias negativas y cuando no se satisface las necesidades, es donde se origina los llamados esquemas desadaptados, los cuales son representaciones disfuncionales almacenado en la memoria de las personas, conformadas por emociones, pensamientos y sensaciones asociadas a uno mismo y a los demás (Sánchez, Andrade y Gómez, 2019). Sobre ello, los esquemas han sido ampliamente estudiados por diversos autores de la psicología cognitiva como Frederick Bartlett, Jean Piaget, Jerome Bruner, Aaron Beck, en otros; quienes influenciaron en Jeffrey Young, para desarrollar su teoría denominada Esquemas Desadaptados Tempranos, donde se postuló que estos esquemas se originan en la infancia y tienden a persistir durante toda la vida, determinando como la persona piensa, siente y actúa, los mismos que dan origen a una serie de problemáticas como trastornos de personalidad, del estado de ánimo, conductas de riesgo y en general afectan el bienestar de las personas (Pérez y Torres, 2016).

Desde la perspectiva teórica de Young, se plantean 18 esquemas que están organizados en 5 dominios, las cuales están asociadas a necesidades no satisfechas y a relaciones disfuncionales ocurridas en la primera infancia (Lyrakos, 2014). El primer dominio es la desconexión y rechazo, el cual está compuesto por los esquemas de abandono, desconfianza, privación emocional, 
imperfección y aislamiento social; el segundo dominio denominado deterioro de la autonomía y desempeño insuficiente, lo conforman los esquemas de dependencia, vulnerabilidad, apego inmaduro y fracaso; el tercer dominio se refiere a los limites deficitarios, compuesto por los esquemas grandiosidad e insuficiente autocontrol; el cuarto dominio dirigido a las necesidades de los demás, lo conforman los esquemas subyugación, autosacrificio y búsqueda de aprobación; finalmente el ultimo dominio es la sobrevigilancia e inhibición, conformada por los esquemas de normas inalcanzables, negativismo, inhibición emocional y castigo (Young y Klosko 2012).

Es en base a estos esquemas desadaptados las personas desarrollan comportamientos y estilos de afrontamientos disfuncionales, los cuales surgen como respuesta de las ideas construidas y elaboradas en etapas tempranas (Bach, et al., 2016). Es decir, el esquema funciona como un impulsador de las conductas, pensamientos o manifestaciones emocionales, las cuales pueden ser manifestadas en diversas etapas de la vida de la persona, siendo sostenidos en el tiempo.

Los esquemas desadaptativos tempranos al ser estables y duraderos, generan diversas dificultades en la vida de las personas. Dentro de la población clínica diversos autores lo han relacionado los esquemas desadaptativos a la manifestación de trastornos de personalidad (Millon y Davis 1998; Young, et al., 2013; Petrocelli et al., 2001; Bahramizadeh, 2011; Montesinos, 2018), a trastornos de depresión y ansiedad (Agudelo, Casadiegos y Sánchez, 2009; Cavete, et al., 2013; Posada y Villa, 2016; Ramírez, 2017, Sánchez, et al., 2019), también se ha asociado a las adicciones (Shaghaghy et al., 2011; Malacas, 2013; Chávez, 2019), para los cuales se ha diseñado diversos tratamientos en base a la Teoría de Esquemas. Sin embargo, los esquemas desadaptativos también originan en las personas pensamientos, comportamientos y sentimientos que generan malestar, que no necesariamente satisfacen los criterios diagnósticos para un trastorno psicológico como tal, pero que representan dificultades en la vida de los individuos.

De lo mencionado, en la presente investigación se pretende establecer la relación entre los esquemas desadaptativos tempranos y el bienestar psicológico en pacientes que acuden a consulta externa en una Institución Prestadora de Servicios de Salud de Lima. Al respecto, el bienestar psicológico es una variable bastante amplia porque describe aspectos subjetivos, conductuales, emocionales y sociales, vinculado a la manera como las personas se enfrentan a las demandas del entorno (Freire et al., 2017). Para ello, se asume el bienestar psicológico desde la teoría multidimensional de Ryff, la cual lo describe como la satisfacción que un individuo experimenta 
sobre sí mismo, siendo resultado de la valoración sobre sus características, desempeño, logros, relaciones y otros aspectos de su vida (Casullo, 2006). En ese sentido, desde esta teoría las personas con alto bienestar, se caracterizan por tener una valoración positiva de su vida, poseen elevados niveles de optimismo, mayor sentido por la vida, altos grado de motivación, preponderancia de sentimientos positivos, logrando una mayor funcionamiento psicológico (Diener, et al., 2009).

\section{Marco teórico}

El modelo multidimensional del bienestar psicológico, fue desarrollado por Carol Ryff, quien sostuvo que el bienestar está asociado a la búsqueda de la autorrealización, del desarrollo de las capacidades, habilidades, del desarrollo personal (Ryff, 2014). Dichos planteamientos de Ryff fueron influenciados por las propuestas de Maslow y Rogers, sosteniendo que el bienestar de las personas surge por el esfuerzo que estas realizan para alcanzar el desarrollo de su potencial, lo cual provee una mayor experimentación de pensamientos, emociones y sentimientos positivos hacia la vida. Por lo tanto, representa una postura eudamónica del bienestar, explicando que este grado de satisfacción va más allá del placer sensorial que un individuo puede experimentar (Huta y Waterman, 2014).

El bienestar psicológico desde la teoría de Ryff está compuesto por seis factores o dimensiones: La autoaceptación, el desarrollo personal, sentido de vida, autocontrol, vínculos y autonomía (Ryff, 2014). La autoaceptación describe el grado de bienestar acerca de las características, recursos y limitaciones individuales; el desarrollo personal, describe el bienestar entorno a su mejoramiento de las capacidades, habilidades y actitudes de uno mismo; el sentido de vida, es el bienestar acerca de los objetivos, metas, logros y dirección que tiene las personas; la dimensión control, describe el bienestar relacionado a la autorregulación del individuo frente a las diferentes situaciones proveyendo equilibrio; la dimensión vínculos, representa el bienestar que tiene las personas en relación a la capacidad para lograr relaciones satisfactorias con los demás; finalmente, la autonomía, describe a la capacidad que tienen los individuos para con independencia tanto para actuar como también para tomar decisiones (Casullo, 2006).

Sobre el bienestar psicológico, diversos autores como Castro (2009), Reise, Bonifav y Haviland (2013) y Sagone y De Caroli (2014) sostuvieron que representa un constructo donde interactúan diferentes factores, los cuales agrupándolo se pueden tener dos principales: factores ambientales y factores individuales. Sobre los factores ambientales, se tiene las condiciones de 
vida, oportunidades de desarrollo, estimulación de las capacidades, reglas, expectativas, metas generadas, rutinas saludables, seguridad, apoyo y refuerzo positivo. Por otro lado, los factores individuales están las características de personalidad, disposiciones emocionales, niveles de energía, grado de adaptabilidad, aptitudes específicas, estrategias de afrontamiento, entre otras.

Por consiguiente, en la presente investigación se realiza un análisis cuantitativo sobre la relación existente entre los esquemas desadaptativos tempranos y bienestar psicológico en pacientes de un centro de salud, con el propósito de diseñar propuestas de mejora para una población con características específicas. Para ello, el objetivo de estudio fue determinar si los esquemas desadaptativos tempranos se relacionan de manera significativa con el bienestar psicológico de los pacientes que acuden a consulta externa en una Institución Prestadora de Servicios de Salud - IPRESS, Lima, 2020.

\section{Método}

La investigación corresponde al enfoque cuantitativo, donde se utilizó la medición numérica de las variables y un análisis estadístico para llegar a las conclusiones. Es un tipo de estudio básico debido a que la presente investigación estuvo orientada a realizar un aporte teórico, es decir aportar información estructurada acerca de las variables de estudio, dicha información será utilizada para una futura aplicación práctica (Valderrama, 2013). El diseño fue no experimental, porque en la investigación no se aplicó ningún programa o tratamiento con la intención de alterar el estado de las variables, solo se realizaron mediciones para obtener datos, los cuales fueron analizados y permitieron explicar el comportamiento de los fenómenos de estudio en un determinado contexto. En nivel de investigación se denominó correlacional, porque el propósito fue establecer la relación entre dos variables en una muestra especifica (Hernández y Mendoza, 2018).

En el estudio participaron 120 pacientes de una institución prestadora de servicios de salud (IPRESS) de Lima, la selección de los mismos se realizó utilizando procedimientos no probabilísticos, siendo la muestra de tipo censal. Para la recolección de información sobre las variables de interés se utilizó la encuesta y como instrumentos se utilizó la Escala de esquemas de Young (YSQ-SF) (2017) y la Escala de Bienestar psicológico Bieps-A de Casullo. 
La escala YSQ-SF fue adaptada al medio en una muestra de estudiantes universitarios por Iturregui (2017), donde se determinó la validez de contenido mediante criterio de cinco expertos en el tema; la validez de constructo, dio como resultado valores de KMO: 0.809, X2 = 7769.619 Gl. 2775 Sig. 0.000. En cuanto a la confiabilidad, en el estudio de Iturregui (2017) se obtuvo un índice de alfa de Cronbach de 0,98 para la escala total y valores superiores a 0,86 para cada dimensión. En el presente estudio, la escala se sometió a criterio de tres jueces, cuyos resultados determinaron su aplicabilidad y se aplicó una prueba piloto a 30 pacientes que acuden a consulta externa en una Institución Prestadora de Servicios de Salud - IPRESS de Lima, donde se obtuvo un índice de confiabilidad mediante consistencia interna de 0,89 , el cual refleja una alta confiabilidad del instrumento.

La Escala de Bienestar Psicológico Bieps-A fue adaptada al medio en una muestra de docentes del nivel secundario por Segura (2017) donde se sometió al instrumento a criterios de cinco expertos, cuyos resultados indicaron su aplicabilidad (V de Aiken=1.00); por otro lado, Segura (2017) determinó que la confiabilidad de la escala es de 0,950 mediante el estadístico de alfa de Cronbach. En el presente estudio, el instrumento se sometió a criterio de tres jueces expertos, cuyos resultados determinaron su aplicabilidad; así mismo, se aplicó una prueba piloto a 30 pacientes que acuden a consulta externa en una Institución Prestadora de Servicios de Salud - IPRESS de Lima, donde se obtuvo un alfa de Cronbach de 0,82, el cual indica que el instrumento tiene una alta confiabilidad. Por ello, en la investigación se siguió el siguiente procedimiento:

En primer lugar, se solicitó la autorización de la IPRESS para la realización de la investigación, brindando información referente a los objetivos y la finalidad de la misma. Luego se procedió a seleccionar a los participantes de manera no probabilística - intencional, teniendo en cuenta su disponibilidad y participación voluntaria, para ello se les brindó un documento denominado consentimiento informado, el cual fue firmado por los pacientes como señal de aceptación.

En segundo lugar, se procedió a seleccionar los instrumentos de recolección de datos, considerando su validez y confiabilidad. Posterior a ello, se procedió a recabar información mediante la aplicación de los cuestionarios a los pacientes que aceptaron participar en la investigación, dicho procedimiento tuvo una duración de 20 minutos aproximadamente. En tercer lugar, se procedió a elaborar una base de datos con la información recolectada y se procedió 
analizar los datos en el programa estadístico SPSS V26. Dichos resultados fueron interpretados y luego se elaboró las conclusiones del estudio.

\section{Resultados}

\section{Datos sociodemográficos}

\section{Tabla 1}

Datos sociodemográficos de los participantes

\begin{tabular}{llcc}
\hline & & Frecuencia & Porcentaje \\
\hline Edad & $18-25$ & 18 & $15,0 \%$ \\
& $26-40$ & 49 & $40,8 \%$ \\
& 41 a 60 & 25 & $20,8 \%$ \\
& 61 a más & 28 & $23,3 \%$ \\
Sexo & Femenino & & \\
& Masculino & 78 & $65,0 \%$ \\
& & 42 & $35,0 \%$ \\
Estado civil & Soltero(a) & & \\
& Casado(a) & 41 & $34,2 \%$ \\
& Divorciado(a) & 47 & $39,1 \%$ \\
& Viudo(a) & 14 & $11,7 \%$ \\
& & 18 & $15,0 \%$ \\
\hline
\end{tabular}

En la tabla 1, se presentan los datos sociodemográficos de 120 pacientes de una IPRESS de Lima. En cuanto a la edad, el 40,8\% se encuentra en el rango de 26 a 40 años, un 23,3\% se ubica en el rango de 61 a más, el 20,8\% posee entre 41 a 60 años y solo el 15\% tiene entre 18 a 25 años. En lo respecta al sexo de los participantes, el $65 \%$ es de sexo femenino y el $35 \%$ son masculinos. En relación al estado civil, el 39,1\% son casados, los 34,2\% solteros, un $15 \%$ son viudos y un $11,7 \%$ son divorciados.

\section{Relación entre las variables y dimensiones}

Los resultados presentados fueron analizados utilizando estadísticos no paramétricos (Rho Spearman), en vista de que las variables son de naturaleza cualitativa. 


\section{Tabla 2}

Coeficientes de correlación para las variables y dimensiones

\begin{tabular}{|c|c|c|c|}
\hline & & & Bienestar psicológico \\
\hline Rho de & Esquemas desadaptativos & Coeficiente de correlación &,$- 764^{* *}$ \\
\hline \multirow[t]{17}{*}{ Spearman } & tempranos & Sig. (bilateral) &, 000 \\
\hline & & $\mathrm{N}$ & 120 \\
\hline & Desconexión/rechazo & Coeficiente de correlación &,$- 617^{* *}$ \\
\hline & & Sig. (bilateral) &, 000 \\
\hline & & $\mathrm{N}$ & 120 \\
\hline & Autonomía/desempeño & Coeficiente de correlación &,$- 623^{* *}$ \\
\hline & & Sig. (bilateral) &, 000 \\
\hline & & $\mathrm{N}$ & 120 \\
\hline & Limites inadecuados & Coeficiente de correlación &,- 166 \\
\hline & & Sig. (bilateral) & ,069 \\
\hline & & $\mathrm{N}$ & 120 \\
\hline & Dirigido hacia el otro & Coeficiente de correlación &,$- 249^{* *}$ \\
\hline & & Sig. (bilateral) & ,006 \\
\hline & & $\mathrm{N}$ & 120 \\
\hline & Sobrevigilancia/inhibición & Coeficiente de correlación &,$- 424^{* *}$ \\
\hline & & Sig. (bilateral) &, 000 \\
\hline & & $\mathrm{N}$ & 120 \\
\hline
\end{tabular}

**. La correlación es significativa en el nivel 0,01 (bilateral).

En la tabla 2, se presentan las correlaciones entre los esquemas desadaptativos con el bienestar psicológico en una muestra de 120 pacientes de una IPRESS de Lima, donde se aprecia que los esquemas desconexión/rechazo (-0,617), autonomía/desempeño $(-0,623)$, dirigido hacia el otro $(-0,249)$ y sobrevigilancia $(-0,424)$, presentan una relación significativa $($ sig. $<0,05)$ e inversa con el bienestar psicológico. Por otro lado, entre el esquema limites inadecuados y bienestar psicológico no se encontraron relaciones estadísticamente significativas (sig.>0,05). 


\section{Relación entre los esquemas desadaptativos tempranos y el bienestar psicológico de los pacientes}

\section{Tabla 3}

Coeficientes de correlación entre los esquemas desadaptativos y el bienestar psicológico

\begin{tabular}{lllc}
\hline & & \multicolumn{1}{c}{$\begin{array}{c}\text { Bienestar } \\
\text { psicológico }\end{array}$} \\
\hline Rho de & Esquemas desadaptativos & Coeficiente de \\
Spearman & correlación & $-0,764$ \\
& tempranos & Sig. (bilateral) & 0,000 \\
& $\mathrm{~N}$ & 120 \\
\hline
\end{tabular}

En la tabla 3, se describen los coeficientes de correlación para los esquemas desadaptativos tempranos y el bienestar psicológico en una muestra de 120 pacientes de una IPRESS de Lima. Del análisis, se observa que entre las variables existe un valor de Rho=-0,764, el cual indica una correlación negativa (inversa), con una intensidad categorizada como muy fuerte (Hernández y Mendoza, 2018). Así mismo, se observa un valor $\mathrm{p}=0,000$ (significancia) inferior a 0,05, explicando que entre las variables la relación es significativa. Estos resultados permiten aceptar la hipótesis de estudio, es decir los esquemas desadaptativos tempranos presentan una relación significativa e inversa con el bienestar psicológico en pacientes que acuden a consulta externa en una Institución Prestadora de Servicios de Salud - IPRESS, Lima, 2020.

\section{Relación entre la dimensión desconexión/rechazo y el bienestar psicológico de los pacientes}

\section{Tabla 4}

Coeficientes de correlación entre la dimensión desconexión/rechazo y el bienestar psicológico

\begin{tabular}{llc}
\hline & & Bienestar psicológico \\
\hline & Coeficiente de correlación & $-0,617$ \\
Rho de Spearman Desconexión/ rechazo & Sig. (bilateral) & 0,000 \\
& N & 120 \\
\hline
\end{tabular}

En la tabla 4, se presentan los coeficientes de correlación para los esquemas desconexión/rechazo y el bienestar psicológico en una muestra de 120 pacientes de una IPRESS 
de Lima. Donde se aprecia un valor de correlación Rho=-0,617 el cual describe una relación negativa (inversa) entre las puntuaciones analizadas, con un intensidad considerable (Hernández y Mendoza, 2018). Del mismo modo, se observa un coeficiente de significancia de $\mathrm{p}=0,000$, indicando que la relación entre las variables es significativa $(\mathrm{p}<0,05)$. Los resultados permitieron demostrar que los esquemas desadaptativos tempranos desconexión/rechazo presentan una relación significativa e inversa con el bienestar psicológico en pacientes que acuden a consulta externa en una Institución Prestadora de Servicios de Salud - IPRESS, Lima, 2020.

\section{Relación entre los esquemas autonomía/desempeño y el bienestar psicológico de los pacientes}

\section{Tabla 5}

Coeficientes de correlación entre los esquemas autonomía/desempeño y el bienestar psicológico

\begin{tabular}{|c|c|c|}
\hline & & Bienestar psicológico \\
\hline \multirow{3}{*}{ Rho de Spearman Autonomía/ desempeño } & Coeficiente de correlación & $-0,623$ \\
\hline & Sig. (bilateral) & 0,000 \\
\hline & $\mathrm{N}$ & 120 \\
\hline
\end{tabular}

En la tabla 5, se describen los coeficientes de correlación para los esquemas autonomía/desempeño y el bienestar psicológico en una muestra de 120 pacientes de una IPRESS de Lima. Donde se puede apreciar un valor de Rho=-0,623, el cual indica una correlación negativa (inversa), con una intensidad categorizada como considerable (Hernández y Mendoza, 2018). Además, se observa un valor de significancia $\mathrm{p}=0,000$, explicando que entre las puntuaciones analizadas existe una relación significativa $(\mathrm{p}<0,05)$. El análisis permitió aceptar la hipótesis de estudio, demostrando que los esquemas desadaptativos tempranos autonomía/desempeño presentan una relación significativa e inversa con el bienestar psicológico en pacientes que acuden a consulta externa en una Institución Prestadora de Servicios de Salud - IPRESS, Lima, 2020. 


\section{Relación entre los esquemas límites inadecuados y el bienestar psicológico de los pacientes}

\section{Tabla 6}

Coeficientes de correlación entre los límites inadecuados y el bienestar psicológico

\begin{tabular}{|c|c|c|}
\hline & & Bienestar psicológico \\
\hline & Coeficiente de correlación & $-0,166$ \\
\hline Rho de Spearman & Sig. (bilateral) & 0,069 \\
\hline & $\mathrm{N}$ & 120 \\
\hline
\end{tabular}

En la tabla 6, se presentan los coeficientes de correlación para los esquemas límites inadecuados y el bienestar psicológico en una muestra de 120 pacientes de una IPRESS de Lima. Donde se observa que las puntuaciones analizadas presentan un valor de correlación Rho=-0,166 el cual describe una relación negativa (inversa) con un intensidad categorizada como muy débil (Hernández y Mendoza, 2018). Así mismo, se puede apreciar un coeficiente de significancia de $\mathrm{p}=0,069$, indicando que la relación entre las variables no es significativa ( $p>0,05)$. De lo descrito, se rechaza la hipótesis de estudio, es decir los esquemas desadaptativos tempranos de la dimensión limites inadecuados no presentan una relación significativa con el bienestar psicológico en pacientes que acuden a consulta externa en una Institución Prestadora de Servicios de Salud IPRESS, Lima, 2020.

\section{Relación entre los esquemas tendencia hacia el otro y el bienestar psicológico de los pacientes}

\section{Tabla 7}

Coeficientes de correlación entre los límites inadecuados y el bienestar psicológico

\begin{tabular}{clc}
\hline & & Bienestar psicológico \\
\hline \multirow{2}{*}{ Rho de Spearman Tendencia hacia el otro } & Sig. (bilateral) & $-0,249$ \\
& N & 0,006 \\
& & 120 \\
\hline
\end{tabular}

En la tabla 7, se presentan los coeficientes de correlación para los esquemas tendencia hacia el otro y el bienestar psicológico de los pacientes. Donde se puede observar un valor de Rho=- 
0,249 dicho coeficiente indica que entre las variables existe una relación negativa (inversa) y en un grado muy débil (Hernández y Mendoza, 2018). En cuanto a la significancia entre las variables, se encontró un valor de $\mathrm{p}=0,006$, el cual indica que entre las puntuaciones analizadas existe una relación significativa $(\mathrm{p}<0,05)$. De los resultados analizados, se demostró que los esquemas desadaptativos tempranos de la dimensión tendencia hacia el otro presentan una relación significativa e inversa al bienestar psicológico en pacientes que acuden a consulta externa en una Institución Prestadora de Servicios de Salud - IPRESS, Lima, 2020.

\section{Relación entre los esquemas sobrevigilancia y el bienestar psicológico de los pacientes}

\section{Tabla 8}

Coeficientes de correlación entre los esquemas sobrevigilancia y el bienestar psicológico

\begin{tabular}{lllc}
\hline & & Bienestar psicológico \\
\hline \multirow{3}{*}{ Rho de Spearman } & \multirow{3}{*}{ Sobrevigilancia } & Sig. (bilateral) & $-0,424$ \\
& & $\mathrm{~N}$ & 0,000 \\
& & & 120 \\
\hline
\end{tabular}

En la tabla 8, se describen los coeficientes de correlación para los esquemas sobrevigilancia y el bienestar psicológico en una muestra de 120 pacientes de una IPRESS de Lima. Del análisis, se observa que entre las variables existe un valor de Rho=-0,424, el cual indica una correlación negativa (inversa), con una intensidad categorizada como media (Hernández y Mendoza, 2018). Así mismo, se observa un valor $\mathrm{p}=0,000$ (significancia) inferior a 0,05, explicando que entre las variables la relación es significativa. Estos resultados permiten aceptar la hipótesis de estudio, es decir los esquemas desadaptativos tempranos de la dimensión sobrevigilancia e inhibición presentan una relación significativa e inversa al bienestar psicológico en pacientes que acuden a consulta externa en una Institución Prestadora de Servicios de Salud - IPRESS, Lima, 20120.

\section{Discusión}

Referente al objetivo general, los resultados analizados permitieron determinar que los esquemas desadaptativos tempranos presentan una relación significativa e inversa con el bienestar 
psicológico en un grupo de pacientes que asisten a una IPRESS. Esto permite explicar que los esquemas cognitivos que representan estructuras rígidas e inflexibles, construidas desde los primeros años de vida en relación a la necesidades emocionales no satisfechas de los pacientes (Young, et al. 2013) se relacionan de manera inversa con el grado de satisfacción que los pacientes experimentan sobre sí mismos, en base a la valoración de sus características, logros, relaciones y otros aspectos de su vida (Casullo, 2006). De lo mencionado, va en la misma línea que los resultados de Meza y Santayana (2018), De la Fuente (2017) y Carranza (2017) quienes mencionaron que los esquemas desadaptativos están asociados a valoraciones negativas de sí mismo y a una baja satisfacción de las personas. Los hallazgos del estudio permiten explicar que las personas que tienden a presentar puntuaciones altas en los esquemas desadaptativos, a la vez tienden a experimentar un menor nivel de bienestar psicológico.

De manera específica, se determinó que los esquemas desadaptativos desconexión/rechazo presentan una relación significativa e inversa con el bienestar psicológico en pacientes que acuden a consulta externa en una IPRESS. Es decir, que aquellos esquemas que describen a personas que son incapaces de lograr un apego seguro, de establecer vínculos satisfactorios con los demás, considerando sus necesidades de seguridad, protección, pertenencia, estabilidad y amor, no son satisfechas (Young y Kiosko, 2012) se relacionan de manera inversa con el grado de satisfacción que los pacientes experimentan frente a su vida, como resultado de la valoración de sus atributos, capacidades, logros, relaciones, metas y otros aspectos (Casullo, 2006). Los hallazgos concuerdan con lo encontrado por Iturregui (2017) donde se mencionó que el apego seguro es una condición fundamental para lograr adultos con un alto grado de satisfacción personal. Sobre ello, los pacientes que desarrollan estos esquemas pertenecen a familias disfuncionales, expuestos ambientes carentes de afecto, estabilidad y situaciones traumatizantes.

De la misma manera, se demostró que los esquemas desadaptativos tempranos autonomía/desempeño presentan una relación significativa e inversa con el bienestar psicológico en pacientes que acuden a consulta externa en una IPRESS. Es decir el esquema que describe a individuos que no logran una actuación independiente, por no poder desligarse de las figuras parentales (Young y Kiosko 2012) se relacionan de manera inversa con el grado de satisfacción que los pacientes experimentan frente a su vida como resultado de la valoración de sus atributos, capacidades, logros, relaciones, metas y otros aspectos (Casullo, 2006). Los hallazgos son similares 
a lo encontrado por Cárdenas, Neves y Sajuán., (2019) e Iturregui (2017) donde se hizo referencia que la actuación autónoma de las personas, permiten que estas alcancen sus objetivos y contribuye aumentar el grado de bienestar. Al respecto, los pacientes que desarrollan estos esquemas provienen de familias con exceso de sobreprotección, donde no se dada un refuerzo adecuado a las conductas del niño, teniendo una repercusión negativa en su bienestar psicológico en la etapa adulta.

Por otro lado, el análisis indicó que los esquemas desadaptativos tempranos límites inadecuados no presentan una relación significativa con el bienestar psicológico en pacientes que acuden a consulta externa en una IPRESS. Esto quiere decir que el esquema cognitivo que describe a individuos carentes de autocontrol, autodisciplina, reciprocidad y cooperación, con tendencia a no respetar el derecho de los demás y considerarse superior a los demás (Young y Kiosko 2012), no se relaciona de manera significativa al grado de satisfacción que los pacientes experimentan sobre su vida, ambas variables presentan un comportamiento diferente. Estos resultados son similares a lo encontrado por Moyano (2017) donde se sostuvo que los límites que se establecen dentro de los entornos familiares fortalecen la funcionalidad más no necesariamente la satisfacción de la misma. De lo mencionado, los pacientes que presentan estos esquemas provienen de familias donde existe permisividad, donde no se existe sentido de autoridad y límites claros, (Cid, 2016).

Los resultados analizados dieron un explicación que los esquemas desadaptativos tempranos tendencia hacia el otro presentan una relación significativa e inversa al bienestar psicológico en pacientes que acuden a consulta externa en una IPRESS. Explicando que el esquema que describe personas que están enfocadas a satisfacer a los demás, sin importa el bienestar propio, estas comportamientos se realizan con la finalidad de lograr la aprobación de los demás y no perder los vínculos (Young y Kiosko 2012), se relaciona de manera inversa con el grado de satisfacción que los pacientes experimentan hacia su vida. Los resultados encontrados concuerdan con los estudios de Ponce, Aiquipa y Arboccó (2019) y Holguín (2018) quienes concluyeron que la esquematización del otro como prioridad, está relacionado a estados bajos de satisfacción personal. Estos esquemas son generados en pacientes que pertenecen a familias donde se valora mucho las apariencias y las expectativas sociales antes que las propias necesidades del niño, repercutiendo en su propia valoración de su vida. 
Se demostró que los esquemas desadaptativos tempranos de la dimensión sobrevigilancia e inhibición presenta una relación significativa e inversa al bienestar psicológico en pacientes que acuden a consulta externa en una IPRESS. Es decir que el esquema que describe a personas que se esfuerzan en controlar y reprimir sus emociones, buscando de manera excesiva no cometer errores a coste de la satisfacción y bienestar de uno mismo (Young y Kiosko 2012), se relaciona de manera inversa con el grado de satisfacción que los pacientes experimentan hacia su vida, como resultado de la valoración de sus características, metas, vínculos, proyectos y otros aspectos. Estando en la misma dirección los estudios de Sánchez, et al., (2019) y Meza y Santayana (2018) quien sostuvieron que la inadecuada regulación emocional, está asociado a niveles altos de ansiedad, depresión y menor satisfacción por la vida. Estos esquemas se generan porque la espontaneidad y la libertad para el juego fueron restringidas, poniendo un excesivo énfasis en la libre expresión, estas personas provienen de familias demandantes, exceso de deberes y de control de la conducta.

Finalmente, si bien los hallazgos del estudio confirman la relación entre los esquemas desadaptativos y el bienestar psicológico, estos representan un aporte teórico que permite describir el comportamiento de las variables en esta población, mas no explica una relación de causalidad entre las mismas. Los resultados representan una base sobre la cual se deben realizar otros estudios que permitan respaldar las conclusiones encontradas y realizar un análisis a mayor profundidad (explicativo causal) de las variables, obteniendo evidencia sólida que permita proponer intervenciones desde la terapia de esquemas con la finalidad de mejorar el bienestar y la calidad de vida de este grupo poblacional.

\section{Conclusiones}

Los resultados encontrados permiten concluir que los esquemas desadaptativos tempranos presentan una relación significativa e inversa con el bienestar psicológico en pacientes que acuden a consulta externa en una IPRESS de Lima, lo cual permite explicar que a mayor presencia de esquemas desadaptativos, los pacientes tienden a experimentar menores niveles de bienestar psicológico, por lo contrario aquellos personas que tienen menores puntuaciones en los esquemas desadaptativos tienden a experimentar mayor bienestar.

Del análisis por dimensiones, se determinó que los esquemas desadaptativos tempranos desconexión, autonomía, tendencia hacia el otro y sobrevigilancia presentan una relación significativa e inversa con el bienestar psicológico en pacientes que acuden a consulta externa en 
IPRESS de Lima; sin embargo, el esquema limites inadecuados no presentan una relación significativa con el bienestar psicológico en dicho grupo poblacional.

\section{Referencias}

Agudelo, D., Casadiegos, C. y Sanchez, D. (2009). Relación entre esquemas maladaptativos tempranos y características de ansiedad y depresión en estudiantes universitarios. $\begin{array}{lllllll}\text { Universitas } & \text { Psychologica, } & 8 & (1), & 87 & - & 103 .\end{array}$ http://www.scielo.org.co/pdf/rups/v8n1/v8n1a7.pdf

Bach, B., Simonsen, E., Christoffersen, P. \& Kriston, L. (2015). The Young Schema Questionnaire 3 Short Form (YSQ-S3): Psychometric properties and association with personality disorders in a Danish mixed sample. European Journal of Psychological Assessment, 33, 134 - 143. https://doi.org/10.1027/1015-5759/a000272

Bahramizadeh, H. (2011). Early Maladaptive schemas and agreeablenes in personality five factor model. Procedia - Social and Behavioral Sciences, 30, 548-551. https://doi.org/10.1016/j.sbspro.2011.10.107

Calvete, E., Orue, I., \& Hankin, B. L. (2013). Early maladaptive schemas and social anxiety in adolescents: The mediating role of anxious automatic thoughts. Journal of Anxiety Disorders, 27, 278-288. https://doi.org/10.1016/j.janxdis.2013.02.011

Cárdenas, V., Neves, D. y Sanjuán, M. (2019). Esquemas cognitivos y codependencia emocional en mujeres de 21 a 46 años del asentamiento urbano caracoles de la ciudad de Cúcuta. (Tesis de licenciatura, Universidad Simón Bolívar). https://bonga.unisimon.edu.co/bitstream/handle/20.500.12442/6082/PDF_Resumen.pdf?s equence $=1$

Carranza, F. (2017). Esquemas maladaptativos tempranos y autoestima en adolescentes mujeres de una institución educativa de Lima. (Tesis de licenciatura, Universidad Alas Peruanas). http://repositorio.uap.edu.pe/handle/uap/81?offset=180

Castro, A. (2009). Bienestar psicológico: cuatro décadas. Revista interuniversitaria de formación del profesorado, 66 (23), 43 -72. https://www.redalyc.org/pdf/274/27419066004.pdf

Casullo, M. (2006). Evaluación del Bienestar Psicológico en Iberoamérica. Buenos Aires: Paidos.

Chávez, M. (2019). Estructuras psicológicas tempranas, impulsividad, adicciones a sustancias y comportamentales en jóvenes de Ecuador. (Tesis doctoral, Universidad Complutense de Madrid). https://eprints.ucm.es/55627/1/T41148.pdf

Cid, J. (2016). El esquema como organizador de la personalidad y sus trastornos: Estudio psicométrico de la adaptación española del Young Schema Questionnaire-Short Form (Tesis doctoral, Universidad Autónoma de Barcelona). http://hdl.handle.net/10803/370109 
De la Fuente, M. (2017). Relación de los Esquemas Mentales desadaptativos y la satisfacción marital en la relación de pareja en la Institución Educativa Particular De La Salle, Arequipa - 2016. (Tesis de maestría, Universidad Católica de Santa María). http://tesis.ucsm.edu.pe/repositorio/handle/UCSM/5962

Diener, E., Wirtz, D., Biswas-Diener, R., Tov, W., Kim-Prieto, C., Choi, D. \& Oishi, S. (2009). New measures of well-being. The Netherlands: Springer.

Freire, C., Ferradás, M., Núñez, J. y Valle, A. (2017). Estructura factorial de las escalas de bienestar psicológico de Ryff en estudiantes universitarios. European Journal of Education and Psychology, 10 (1), 1-8. https://doi.org/10.1016/j.ejeps.2016.10.001

Hernández, R. y Mendoza, C (2018). Metodología de la investigación, las rutas cuantitativa, cualitativa y mixta. México: Mc Graw Hill

Holguín, J. (2018). Esquemas maladaptativos tempranos e insatisfacción corporal en estudiantes de una institución educativa nacional de Trujillo. (Tesis de licenciatura, Universidad Privada del Norte). http://hdl.handle.net/11537/13260

Huta, V. y Waterman, A. (2014). Eudaimonia and its distiction from hedonia: developing a classification and terminology for understanding conceptual and operational definitions. Journaly of Happiness Studies, 15 (6), 1425-1456. https://doi.org/10.1007/s10902-0139485-0

Iturregui, C. (2017). Esquemas disfuncionales tempranos y dependencia emocional en estudiantes universitarios de Lima Norte. (Tesis de maestría, Universidad Peruana Cayetano Heredia). http://repositorio.upch.edu.pe/handle/upch/3787

Lyrakos, D. (2014). The validity of Young Schema Questionnaire 3rd Version and the Schema Mode Inventory 2nd Version on the greek population. Psychology. 3 (12), 1110-1115. http://dx.doi.org/10.4236/psych.2014.55056

Malacas, C. (2013). Esquemas disfuncionales tempranos en consumidores de sustancias psicoactivas internados en comunidades terapéuticas. (Tesis de licenciatura, Universidad Nacional Federico Villarreal). http://repositorio.unfv.edu.pe/handle/UNFV/1069

Meza, K. y Santayana, R. (2018). Esquemas maladaptativos tempranos y depresión en pacientes de una institución de salud de Lima. (Tesis de licenciatura, Universidad Femenina del Sagrado Corazón). http://hdl.handle.net/20.500.11955/428

Millon, T. y Davis, R. (1998). Trastornos de la personalidad, Más allá del DSM-IV. Barcelona: Masson.

Montesinos, N. (2018). Esquemas desadaptativos y rasgos patológicos de la personalidad en pacientes con trastorno por consumo de sustancia. (Tesis de licenciatura, Universidad de Azuay). http://dspace.uazuay.edu.ec/handle/datos/8633 
Moyano, M. (2017). Los esquemas dasadaptativos tempranos, satisfacción familiar y conductas delictivas. (Tesis de licenciatura, Universidad de Aconcagua). http://bibliotecadigital.uda.edu.ar/756

Pérez, L. y Torres, A. (2016). Desarrollo de los esquemas maladaptativos tempranos en madres adolescentes de un CAR. Avances de psicología, 24 (2), 167-174. https://doi.org/10.33539/avpsicol.2016.v24n2.152

Petrocelli, J., Glaser, B., Calhoun, G. y Campbell, L. (2001). Early Maladaptative schemas of personality disorder subtypes. Journal of Personality Disorders, 15 (6), 546-559. https://doi.org/10.1521/pedi.15.6.546.19189

Ponce, C., Aiquipa, J. y Arboccó, M. (2019). Dependencia emocional, satisfacción con la vida y violencia de pareja en estudiantes universitarios. Propósitos y Representaciones, 7. http://dx.doi.org/10.20511/pyr2019.v7nSPE.351

Posada, J. y Villa, A. (2016). Esquemas maladaptativos tempranos y trastornos de ansiedad en la primera infancia y la niñez. Revista Katharsis, 21, 45-68. https://doi.org/10.25057/25005731.762

Ramírez, R. (2017). Esquemas desadaptativos en estudiantes con sintomatología depresiva de un colegio municipal. (Tesis de licenciatura, Universidad Central del Ecuador). http://www.dspace.uce.edu.ec/handle/25000/14622

Reise, S., Bonifay, W. y Haviland, M. (2013). Scoring and modeling psychological measures in the presence of multidimensionality. Journal of Personality Assessment, 95 (2), 129-140. https://doi.org/10.1080/00223891.2012.725437

Ryff, C. (2014). Psychological well-being revisited: advances in the science and practice of eudaimonia. Psychotherapy and Psychosomatics, $83 \quad$ (1), 10-28. https://doi.org/10.1159/000353263

Sagone, E., \& De Caroli, M. (2014). Relationships between psychological well-being and resilience in middle and late adolescents. Procedia-Social and Behavioural Sciences, 141, 881-887. https://doi.org/10.1016/j.sbspro.2014.05.154

Sánchez, A., Andrade, P. y Gómez, M. (2019). Esquemas desadaptativos tempranos y ansiedad en escolares de México. Revista de Psicología Clínica con Niños y Adolescentes, 6 (2), 15-21. http://doi.org/10.21134/rpcna.2019.06.2.2

Segura, N. (2017). Bienestar psicológico en docentes de las instituciones educativas públicas y particulares de nivel secundario del distrito de Los Olivos, 2017. (Tesis de licenciatura, Universidad César Vallejo). http://repositorio.ucv.edu.pe/handle/UCV/11359?localeattribute $=\mathrm{es}$

Shaghaghy, F., Saffarinia, M., Iranpoor, M. y Soltanynejad, A. (2011). The relationship of early maladaptive schemas, atributional styles and learned helplessness among addicted and Non- 
addicted Men. Addict \& Health, $3 \quad$ (2), 45-52. https://www.ncbi.nlm.nih.gov/pmc/articles/PMC3905522/

Valderrama, S. (2013). Pasos para elaborar proyectos y tesis de investigación Científica cuantitativa, cualitativa y mixta. (2a ed.). Lima: San Marcos.

Young, J. y Klosko, J. (2012). Reinventa tu vida. Barcelona: Paidos.

Young, J., Klosko, J., y Weishaar, M. (2013). Terapia de Esquemas: guía práctica. Bilbao: Desclée De Brouwer, S.A. 